\title{
Tradução e Adaptação Transcultural da Versão Brasileira do Schutte Self-Report Emotional Intelligence Test
}

\author{
Translation and Transcultural Adaptation of \\ the Brazilian Version of the Schutte Self-Report \\ Emotional Intelligence Test
}

\author{
Antonio Toledo Júnior \\ João Gabriel Menezes Ducal \\ Marayra Ines França Coury
}

\section{PALAVRAS-CHAVE}

- Ensino.

- Inteligência Emocional.

- Estudos de Validação.

- Tradução.
INTRODUÇÃO: Estudos recentes demonstram que a inteligência emocional (IE) pode melhorar a educação médica e a prática profissional. Não existem instrumentos de avaliação da IE validados para o português (Brasil). OBJETIVO: Realizar tradução e adaptação transcultural do Schutte Self Report Emotional Intelligence Test (SSEIT) do inglês para o português. MATERIAIS E MÉTODOS: A tradução e adaptação transcultural foi realizada em seis etapas: tradução, sintese, tradução reversa, revisão por comitê, pré-teste e confecção da versão final. As traduçães do inglês para o português foram realizadas por um psicólogo e um professor de inglês brasileiros e fluentes em inglês. A retrotradução foi realizada por duas pessoas nativas de língua inglesa que não conheciam o questionário original. O Comitê de Revisão foi formado pelos autores do estudo. A versão final foi submetida ao teste de Cronbach alfa $\left(C_{a}\right)$ para avaliação da consistência e confiabilidade interna. Foram considerados aceitáveis valores $\geq 0,70$. O pré-teste foi realizado em estudantes de Medicina e médicos residentes, que, além de responderem ao questionário, reescreveram as perguntas em suas próprias palavras. RESULTADOS: A versão traduzida do SSEIT para o português apresentou $100 \%$ de equivalência semântica e idiomática. A comparação das versões retrotraduzidas com o questionário original em inglês apresentou discrepâncias semânticas discretas em quatro questões, que tiveram seus textos ajustados. A versão pré-teste foi aplicada em 41 voluntários, após assinatura do Termo de Consentimento Livre e Esclarecido. Sete questionários foram eliminados por preenchimento incompleto. A análise da transcrição mostrou pequenas discrepâncias nas mesmas questões da retrotradução, que foram novamente ajustadas. $O O C_{a}$ foi de 0,786 para o questionário completo e variou de 0,763 a 0,798 entre as questões. CONCLUSÃO: O SSEIT foi traduzido e adaptado para o português com sucesso e apresentou consistência e validade internas aceitáveis de acordo com o teste de Cronbach alfa. 


\section{KEY-WORDS}

- Teaching.

- Emotional Intelligence.

- Validation Studies.

- Translating.

\section{ABSTRACT}

BACKGROUND: Recent studies showed that emotional intelligence (EI) could improve medical education and professional practice. There were no instruments validated for Brazilian Portuguese to evaluate EI level. OBJECTIVE: To translate and cross-culturally adapt the Schutte Self Report Emotional Intelligence Test (SSEIT) for Brazilian Portuguese. METHODS: The translation and cross-cultural adaptation was performed in six steps: translation, synthesis, back translation, expert committee review, pretesting and elaboration of the final version. A Brazilian psychologist and an English Teacher, both fluent in English, performed the translations from English to Portuguese. Two native English speakers who were not familiar with the questionnaire performed the back translations. The authors composed the expert committee review. The internal consistency and reliability of the final version was tested by Cronbach's alpha $\left(C_{a}\right)$. Values of $\geq 0.70$ were considered acceptable. The pretesting was done with medical students and resident doctors, who answered the test and rewrote the sentences in their own words. RESULTS: The Portuguese SSEIT translated version had 100\% semantic and idiomatic equivalence. The comparison between the back translated versions and the original showed slight semantic discrepancies in four questions, therefore the texts were adjusted. The pretesting version was applied to 41 volunteers, after signing an Informed Consent Form. Seven questionnaires were eliminated as they were not filled out properly. Analyses of the rewritten sentences showed slight discrepancies in the same four questions of the back translations, and were again adjusted. The $C_{a}$ was 0.786 for the whole questionnaire and ranged from 0.763 to 0.798 between the questions. CONCLUSION: The translation and cross-cultural adaptation of the SSEIT for Brazilian Portuguese was a success, and showed acceptable internal consistency and reliability according to Cronbach's alpha test.

Recebido em: 8/5/18

Aceito em: 29/5/18

\section{INTRODUÇÃO}

A inteligência emocional (IE) é um dos temas mais debatidos e investigados atualmente em relação às capacidades e competências intelectuais e sociais de um indivíduo, seja em seu ambiente de trabalho, de estudo ou social. As primeiras referências científicas que introduziram o termo "inteligência emocional" datam de 1960 e eram textos que faziam menção a tratamentos psicoterápicos ${ }^{1}$. Já na década de 1980, com a introdução da ideia de múltiplas formas ou facetas da inteligência de forma geral, a investigação sobre as emoções e como elas se relacionavam com a cognição teve um aumento significativo, atingindo seu pico na década de 1990, com a popularização do tema e um grande crescimento no número de publicações que tentavam conceituar e explorar a importância dessa competência em diferentes situações ${ }^{1}$.

A IE pode ser definida assim ${ }^{1}$ :

A inteligência emocional envolve a capacidade de perceber com precisão, de avaliar e de expressar emoções; a capacidade de perceber e/ou gerar sentimentos quando eles facilitam o pensamento; a capacidade de compreender a emoção e o conhecimento emocional; e a capacidade de controlar emoções para promover o crescimento emocional e intelectual.

Estudos em Medicina têm demonstrado a importância da IE na prática profissional, como no estabelecimento e manutenção de uma boa relação médico-paciente ${ }^{2}$. Além disso, no que tange à importância da IE para a educação e formação médica, Johnson ${ }^{3}$ discutiu em sua revisão a importância, para o médico, da capacidade de avaliar as emoções do paciente para uma melhor coleta de história e um diagnóstico mais preciso. Nesse mesmo estudo ${ }^{3}$, o autor ressalta as estratégias para treinar, desenvolver, testar e avaliar as habilidades emocionais dos estudantes de Medicina, que devem necessariamente ser abordadas de forma longitudinal e integral ao longo do curso, não apenas para fixação, mas também para favorecer a incorporação desses conceitos e habilidades.

Outro estudo recente ${ }^{4}$, que avaliou a influência da IE no desempenho de estudantes de Medicina em atividades cirúrgicas, demonstrou que a capacidade de suportar e responder 
bem ao estresse laboral e ocupacional está ligada à IE do estudante ou do médico. Os autores sugeriram treinar o grupo de habilidades que englobam essa área cognitiva para que os alunos tenham melhores mecanismos de enfrentamento do estresse relacionado ao trabalho.

Ranasinghe et al. ${ }^{5}$, por meio de revisão da literatura, confirmam que um nível mais elevado de IE em estudantes de Medicina está associado a desempenho acadêmico melhor em relação às habilidades clínicas e ao trabalho no decorrer da formação. Embora as emoções sejam conhecidas como fenômenos universais, fatores culturais podem influenciar fortemente os sentimentos que estão sendo experimentados, percebidos e transmitidos no decorrer da vida acadêmica ${ }^{5}$.

A IE pode influenciar a educação médica, contribuindo para o aprendizado sobre o profissionalismo e habilidades de comunicação, permitindo formar médico que lide melhor com as adversidades e dificuldades que fazem parte da prática profissional $^{6}$. A pesquisa sobre IE e educação médica mostra-se relevante pela literatura e pode gerar resultados que tenham impacto na formação do futuro profissional e na qualidade da assistência médica prestada por ele.

Vários testes para avaliação da IE estão disponíveis em português para aplicação em empresas, pesquisas e grupos de trabalho. Porém, a maior parte deles não foi traduzida, adaptada ou validada adequadamente para o português, o que impede sua utilização em pesquisas. $\mathrm{O}$ objetivo deste trabalho é realizar a tradução e adaptação transcultural do questionário Schutte Self Report EI Test (SSEIT) para avaliação da IE do inglês para o português do Brasil.

\section{MATERIAIS E MÉTODOS}

\section{Instrumento}

A revisão da literatura realizada no PubMed com os termos emotional intelligence assessment e no site Consortium for Research on Emotion Intelligence in Organizations (http://www. eiconsortium.org/) permitiu identificar dez instrumentos de avaliação de IE validados em inglês. Optou-se por utilizar o questionário SSEIT por já possuir validação psicométrica, boa consistência interna, ser curto e autoaplicado ${ }^{7}$. Sua adaptação e utilização em português foram autorizadas pela autora.

O SSEIT é um questionário autoaplicado de 33 questões, divididas em quatro domínios: percepção de emoções (PE - 10), manejo das próprias emoções (MPE - 9), manejo das emoções de outros (MEO - 8) e utilização das emoções (UE - 6). As perguntas utilizam uma escala de Likert com cinco opções de resposta: (1) discordo totalmente, (2) discordo parcialmente, (3) nem discordo, nem concordo, (4) concordo parcialmente e (5) concordo totalmente. Para o cálculo do escore final, devem- -se inverter os valores das respostas das questões 5, 28 e 33, e somar os valores das questões. Pode-se calcular também o escore individual para quatro domínios relacionados à inteligência emocional: percepção de emoção (perguntas 5, 9, 15, $18,19,22,25,29,32,33)$, gerenciamento de emoções próprias (itens 2, 3, 10, 12, 14, 21, 23, 28, 31), gerenciando das emoções dos outros (itens 1, 4, 11, 13, 16, 24, 26, 30) e utilização das emoções (itens $6,7,8,17,20$, 27). O escore total varia de 33 a 165. As pontuações mais altas indicam nível de inteligência emocional mais elevado. O tempo de aplicação do questionário varia de cinco a dez minutos ${ }^{8}$.

\section{Tradução e adaptação transcultural}

A tradução e adaptação transcultural é um processo qualitativo, no qual um questionário já validado psicometricamente em determinado idioma é traduzido e adaptado para outro idioma9 . O objetivo desse método é realizar uma tradução validada do artigo de modo a usar os estudos psicométricos feitos na língua original. A tradução e adaptação transcultural pode dispensar a realização de estudos psicométricos no novo idioma ${ }^{9,10}$. O método apresenta seis etapas: (1) tradução, (2) síntese, (3) retrotradução, (4) revisão por comitê, (5) pré-teste e (6) confecção da versão final ${ }^{9,10}$.

Na primeira etapa (tradução), a versão original do questionário foi traduzida do inglês para o português (T1 e T2) de forma independente por dois profissionais brasileiros com fluência em inglês, um psicólogo e um professor de Letras. Na etapa de síntese, os autores sintetizaram T1 e T2 em uma versão única do questionário (T1/T2). Foram avaliadas e corrigidas as discrepâncias semânticas e idiomáticas entre T1 e T2 (Quadro 1). Em seguida, a versão T1/T2 foi retrotraduzida do português para o inglês por dois tradutores independentes, nativos de língua inglesa e fluentes em português, que não conheciam a versão original do questionário. Eles produziram duas versões independentes (RT1 e RT2). Na quarta etapa, os autores compararam as versões RT1 e RT2 com a versão original e a versão síntese (T1/T2) com o objetivo de identificar discrepâncias nas equivalências semântica, idiomática, experiencial e conceitual (Quadro 1). Foram realizados os ajustes necessários na versão T1/T2 com base nas discrepâncias observadas e obteve-se a versão pré-final para realização do pré-teste. O pré-teste (quinta etapa) foi planejado para ser realizado em 30 estudantes de Medicina e médicos residentes, conforme recomendado por Beaton et al. ${ }^{9}$ Além de responderem ao questionário, os participantes reescreveram as perguntas em suas palavras, para nova avaliação das equivalências semântica, idiomática, experiencial e conceitual. 


\section{Quadro 1}

\section{Descrição de equivalência semântica, idiomática, experiencial e conceitual ${ }^{9}$}

\begin{tabular}{|c|c|}
\hline Equivalência & Descrição \\
\hline Semântica & $\begin{array}{l}\text { As palavras têm o mesmo significado? Existem vários significados para o mesmo item? Existem dificuldades } \\
\text { gramaticais na tradução? }\end{array}$ \\
\hline Idiomática & $\begin{array}{l}\text { Coloquialismos são difíceis de traduzir. Pode ser necessário que o Comitê elabore uma expressão equivalente à da } \\
\text { versão original. Por exemplo, a expressão feeling downhearted and blue do questionário SF-36 é difícil de traduzir de } \\
\text { modo geral. O Comitê deve identificar uma expressão de significado similar para a população-alvo. }\end{array}$ \\
\hline $\begin{array}{l}\text { Experiencial ou } \\
\text { vivencial }\end{array}$ & $\begin{array}{l}\text { Os itens devem capturar a experiência da vida cotidiana. Entretanto, em diferentes países e culturas, determinada } \\
\text { tarefa pode não ser vivenciada, mesmo que o item possa ser traduzido. Neste caso, o item do questionário deve ser } \\
\text { substituído por um item similar que realmente seja vivenciado na cultura da população-alvo. Por exemplo, o item "você } \\
\text { tem dificuldade de comer com o garfo?" não seria aplicável a uma comunidade ou população que não utiliza talheres } \\
\text { para comer. }\end{array}$ \\
\hline Conceitual & $\begin{array}{l}\text { Algumas palavras podem ter diferentes significados conceituais entre diferentes culturas. Por exemplo, na expressão } \\
\text { "ver sua família com a frequência que você gostaria", a palavra "família" pode ter conceitos diferentes, de acordo com a } \\
\text { cultura, de "o núcleo familiar" ou "toda a família". }\end{array}$ \\
\hline
\end{tabular}

Fonte: Beaton et al. ${ }^{9}$.

Utilizou-se o teste de Cronbach alpha para avaliar a consistência e a validade internas do questionário traduzido. Valores $\geq 0,70$ foram considerados aceitáveis. Para se avaliar a influência de cada questão sobre a consistência interna do questionário, o teste foi calculado excluindo cada uma das questões individualmente.

\section{RESULTADOS}

Tradução e adaptação transcultural

No processo de tradução do questionário original do inglês para o português, o Comitê considerou que houve equivalência semântica e idiomática em 100\% das questões. Após a retrotradução, a comparação entre a versão original em inglês e as duas versões retrotraduzidas mostrou discrepâncias semânticas discretas nas questões 6, 25, 27 e 32 (Quadro 2). Na questão 33, embora não tenha havido discrepância semântica, o Comitê considerou necessário fazer um pequeno ajuste no texto da questão. A equivalência idiomática, experiencial e conceitual foi considerada $100 \%$ entre o questionário original e as versões retrotraduzidas.

\section{Pré-teste}

A versão pré-final do questionário foi aplicada em 41 voluntários, acadêmicos de Medicina e residentes após a assinatura do TCLE. Sete voluntários não preencheram o questionário corretamente e foram eliminados da análise. Dos 34 voluntários restantes, 17 (50,0\%) eram estudantes de Medicina e 18 $(59,2 \%)$ eram mulheres. A idade média deles foi de $26,4( \pm 9,5)$ anos, variando de 19 a 36 anos.

$\mathrm{Na}$ análise qualitativa da transcrição das respostas dos voluntários, observou-se discrepância entre a pergunta e a transcrição em 8,8\% das respostas na questão $6 ; 2,9 \%$ na questão 25;

QUADRO 2
Discrepâncias semânticas encontradas na retrotradução

$5,9 \%$ na questão 27; e 11,8\% na questão 32. Com base nesses resultados, o Comitê fez pequenos ajustes nessas questões. O Quadro 3 apresenta a versão final do questionário em português.

O teste de Cronbach alfa para o questionário completo foi aceitável $(0,786)$. Realizou-se também o teste de Cronbach, retirando-se cada uma das questões, e o resultado se manteve acima de 0,70 (variando de 0,763 a 0,798) em todas as análises (Tabela 1).

O escore médio total foi de 126,6 pontos, o que representa $76,7 \%$ do total possível (Tabela 2). O domínio com maior escore foi o manejo das próprias emoções (37,2 pontos - 82,7\%) e o menor foi percepção das emoções (34,4 pontos - 68,8\%).

Não houve diferenças estatisticamente significativas entre os domínios e no escore total quando se comparou sexo e nível de formação (graduandos versus residentes). 


\section{QUADRo 3
Versão final do questionário em português}

1. Eu sei quando falar sobre meus problemas pessoais com outras pessoas.

2. Quando eu enfrento um problema, lembro-me das ocasiões em que enfrentei problemas semelhantes e consegui resolvê-los.

3. Eu espero me sair bem na maioria das coisas que tento fazer.

4. As pessoas acham fácil confiar em mim.

5. Eu acho difícil entender as mensagens não verbais de outras pessoas.

6. Alguns dos eventos principais de minha vida me levaram a reavaliar o que é importante e o que não é importante para mim.

7. Quando meu humor muda, eu percebo novas possibilidades.

8. Emoções são uma das coisas que fazem minha vida valer a pena.

9. Eu tenho consciência de minhas emoções quando eu as sinto.

10. Eu espero que coisas boas aconteçam.

11. Eu gosto de compartilhar minhas emoções com outras pessoas.

12. Quando eu experimento uma emoção positiva, eu sei como fazêla durar mais.

13. Eu organizo eventos de que outras pessoas gostam.

14. Eu procuro atividades que me fazem feliz.

15. Eu tenho consciência das mensagens não verbais que transmito aos outros.

16. Eu me apresento de maneira que cause boa impressão aos outros.

17. Quando estou de bom humor, acho fácil resolver problemas.

18. Ao olhar a expressão facial das pessoas, eu consigo reconhecer as emoções que elas estão experimentando.

19. Eu sei por que minhas emoções mudam.

20. Quando eu estou de bom humor, eu sou capaz de ter novas ideias.

21. Eu tenho controle sobre minhas emoções.

22. Eu reconheço facilmente minhas emoções quando as vivencio.

23. Eu me motivo quando imagino os bons resultados nas tarefas que assumo.

24. Eu elogio os outros quando fazem algo bem feito.

25. Eu identifico as mensagens não verbais que as outras pessoas enviam.

26. Quando outra pessoa me fala sobre um evento importante em sua vida, eu quase me sinto como se eu tivesse vivido aquilo.

27. Quando sinto uma mudança nas minhas emoções, eu tenho a tendência de ter novas ideias.

28. Quando enfrento um desafio, eu desisto porque acho que vou falhar.

29. Eu sei o que outras pessoas estão sentindo só de olhar para elas.

30. Eu ajudo as pessoas a se sentirem melhor quando elas estão tristes.

31. Eu uso o bom humor para me ajudar a continuar enfrentando os obstáculos.

32. Eu posso dizer como as pessoas estão se sentindo ao ouvir o tom de sua voz.

33. É difícil para mim entender o porquê de as pessoas se sentirem como elas se sentem.

\begin{tabular}{cccccc|}
\multicolumn{7}{c}{ TABELA 1 } \\
Questão & $\begin{array}{c}\text { Análise da consistência e confiabilidade } \\
\text { interna do questionário traduzido } \\
\text { Cronbach } \\
\text { alfa }\end{array}$ & Questão & $\begin{array}{c}\text { Cronbach } \\
\text { alfa }\end{array}$ & Questão & $\begin{array}{c}\text { Cronbach } \\
\text { alfa }\end{array}$ \\
\hline 1 & 0,786 & 12 & 0,779 & 23 & 0,789 \\
\hline 2 & 0,785 & 13 & 0,785 & 24 & 0,783 \\
\hline 3 & 0,793 & 14 & 0,783 & 25 & 0,767 \\
\hline 4 & 0,785 & 15 & 0,778 & 26 & 0,797 \\
\hline 5 & 0,790 & 16 & 0,781 & 27 & 0,792 \\
\hline 6 & 0,795 & 17 & 0,777 & 28 & 0,793 \\
\hline 7 & 0,789 & 18 & 0,781 & 29 & 0,773 \\
\hline 8 & 0,778 & 19 & 0,763 & 30 & 0,787 \\
\hline 9 & 0,780 & 20 & 0,774 & 31 & 0,772 \\
\hline 10 & 0,786 & 21 & 0,779 & 32 & 0,789 \\
\hline 11 & 0,777 & 22 & 0,770 & 33 & 0,798 \\
\hline
\end{tabular}

Cronbach alfa do questionário completo $=0,788$.

\begin{tabular}{|lccc}
\hline \multicolumn{4}{c}{ TABELA 2 } \\
Resultado dos escores por domínio \\
Domínio & $\begin{array}{c}\text { Valor } \\
\text { observado }\end{array}$ & $\begin{array}{c}\text { Desvio } \\
\text { padrão }\end{array}$ & $\begin{array}{c}\text { \% escore } \\
\text { máximo }\end{array}$ \\
\hline Percepção das emoções & 34,4 & $\pm 6,1$ & 68,8 \\
Manejo das próprias emoções & 37,2 & $\pm 4,2$ & 82,7 \\
Manejo das emoções de outros & 31,1 & $\pm 3,7$ & 77,8 \\
Utilização das emoções & 23,9 & $\pm 3,2$ & 79,7 \\
Total & 126,6 & $\pm 12,1$ & 76,7 \\
\hline
\end{tabular}

\section{Aspectos éticos}

O estudo foi aprovado pelo Comitê de Ética em Pesquisa em humanos - Unifenas (CAAE 6507.1617.0.0000.5143). Todos os participantes assinaram o TCLE antes de sua inclusão.

\section{CONCLUSÃO}

O objetivo principal do estudo - fazer a tradução e a adaptação transcultural do questionário SSEIT para o português (Brasil) - foi alcançado com êxito. Durante o processo de tradução e adaptação transcultural, as poucas discrepâncias semânticas e idiomáticas foram corrigidas com sucesso. $\mathrm{O}$ teste de Cronbach alpha mostrou consistência e confiabilidade interna aceitáveis, indicando que a versão em português do SSEIT pode ser utilizada em projetos de pesquisa que envolvam a análise da inteligência emocional em diferentes áreas do conhecimento. 


\section{REFERÊNCIAS}

1. Mayer JD, Roberts RD, Barsade SG. Human abilities: emotional intelligence. Annu Rev Psychol 2008;59:507-36.

2. McLeod SA, Sonnenberg LK. The emotional intelligence of pediatric residents - a descriptive cross-sectional study. Can Med Educ J 2017;8:e44-e51.

3. Johnson DR. Emotional intelligence as a crucial component to medical education. Int J Med Educ 2015;6:179-83.

4. Joseph N. Emotional intelligence and stress in medical students performing surgical tasks. Indian J Public Health 2016;60:166.

5. Ranasinghe P, Wathurapatha WS, Mathangasinghe Y, Ponnamperuma G. Emotional intelligence, perceived stress and academic performance of Sri Lankan medical undergraduates. BMC Med Educ 2017;17:41.

6. Cherry MG, Fletcher I, O'Sullivan H, Dornan T. Emotional intelligence in medical education: a critical review. Med Educ 2014;48:468-78.

7. Schutte NS, Malouff JM, Hall LE, et al. Development and validation of a measure of emotional intelligence. Personality and Individual Differences 1998;25:167-77.

8. Schutte NS, Malouff JM, N B. The Assessing Emotions Scale. In: Stough C, Saklofske D, Parker J, eds. The Assessment of Emotional Intelligence. New York: Springer Publishing; 2009:119-35.

9. Beaton DE, Bombardier C, Guillemin F, Ferraz MB. Guidelines for the process of cross-cultural adaptation of self-report measures. Spine (Phila Pa 1976) 2000;25:3186-91.

10. Sousa VD, Rojjanasrirat W. Translation, adaptation and validation of instruments or scales for use in cross-cultural health care research: a clear and user-friendly guideline. J Eval Clin Pract 2011;17:268-74.

\section{CONTRIBUIÇÃO DOS AUTORES}

Antonio Toledo Júnior: revisão bibliográfica, análise dos dados, redação e revisão do artigo; João Gabriel Menezes Duca: revisão bibliográfica, aplicação dos questionários, banco de dados, análise dos dados, redação e revisão do artigo; Marayra Inez França Coury: aplicação dos questionários, banco de dados, análise dos dados, redação e revisão do artigo.

\section{CONFLITO DE INTERESSES}

Nenhum.

\section{AGRADECIMENTOS}

Os autores agradecem a Profa. Nicola Schutte por permitir a tradução e utilização do questionário e a colaboração do Prof. Virgílio Pereira de Almeida e da psicóloga Estefânia Harsányi pela gentileza de realizar a tradução do questionário do inglês para o português.

\section{ENDEREÇO PARA CORRESPONDÊNCIA}

Antonio Carlos de Castro Toledo Jr.

Rua Califórnia, 570 - apto 501

Sion - Belo Horizonte

CEP 30315-500 - MG

e-mail: antonio.toledo@unifenas.br 